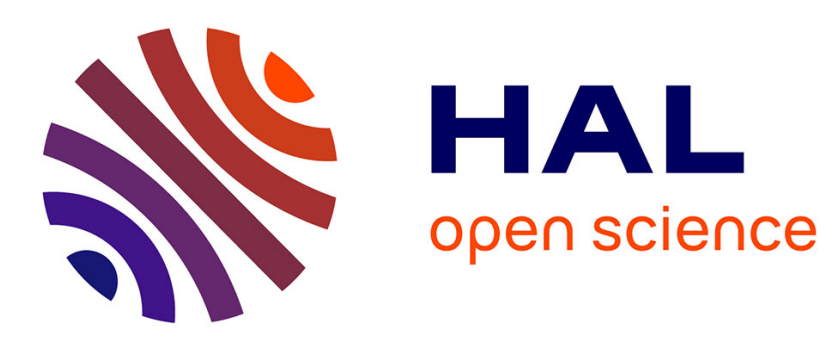

\title{
Optimization of field-free molecular alignment by phase-shaped laser pulses
}

\author{
E. Hertz, A. Rouzee, S. Guerin, B. Lavorel, O. Faucher
}

\section{To cite this version:}

E. Hertz, A. Rouzee, S. Guerin, B. Lavorel, O. Faucher. Optimization of field-free molecular alignment by phase-shaped laser pulses. Physical Review A : Atomic, molecular, and optical physics [1990-2015], 2007, 75, pp.031403. 10.1103/PhysRevA.75.031403 . hal-00397082

\section{HAL Id: hal-00397082 https://hal.science/hal-00397082}

Submitted on 19 Jun 2009

HAL is a multi-disciplinary open access archive for the deposit and dissemination of scientific research documents, whether they are published or not. The documents may come from teaching and research institutions in France or abroad, or from public or private research centers.
L'archive ouverte pluridisciplinaire HAL, est destinée au dépôt et à la diffusion de documents scientifiques de niveau recherche, publiés ou non, émanant des établissements d'enseignement et de recherche français ou étrangers, des laboratoires publics ou privés. 


\title{
Optimization of field-free molecular alignment by phase-shaped laser pulses
}

\author{
E. Hertz, A. Rouzée, S. Guérin, B. Lavorel, and O. Faucher \\ Laboratoire de Physique de l'Université de Bourgogne, \\ UMR CNRS 5027, BP 47870, 21078 Dijon Cedex, France
}

(Dated: January 10, 2007)

\begin{abstract}
We theoretically demonstrate the optimization of field-free molecular alignment by phase-shaped femtosecond laser pulses. The effect is assessed in $\mathrm{O}_{2}$ at $T=60 \mathrm{~K}$ under realistic conditions of intensity and pulse shaping. The spectral laser phase is sampled through 128 control parameters and a self learning evolutionary algorithm combined with a nonperturbative regime calculation is used in order to design the specific phase that maximizes the degree of alignment. The postpulse molecular alignment appears significantly enhanced compared to a Fourier-transform-limited pulse of same energy. The analysis of the target state reveals that the solution is close to the optimal one.

PACS numbers: $42.50 . \mathrm{Hz}, 32.80 . \mathrm{Lg}, 33.80 .-\mathrm{b}$
\end{abstract}

Laser-induced spatial manipulation of molecules has become a subject of growing interests in the last decade covering a range of applications in chemical reactions, surface processing and ultrafast optics [1]. It is now well established that molecules with an anisotropic polarizability exposed to a strong laser pulse experience alignment. In the case of nonresonant short pulses (short with respect to the rotational period), periodic transient molecular alignment takes place after the end of the pulse [2-4] as a result of the subsequent dephasing and rephasing of the rotational wavepacket formed in the vibronic ground state of the molecule. This so-called postpulse molecular alignment is particularly valuable for applications requiring a sample of aligned molecules under fieldfree conditions. In this view, a number of works have focused on the possibility of controlling the dynamics of postpulse alignment. By tailoring the laser pulse shapes, switching of alignment $[5,6]$, control of the rephasing period [7] and control of the transient shapes [8] have been demonstrated. A specific elliptical polarization has been also exploited in order to establish an alternation of alignment along two perpendicular axes [9]. Since the degree of molecular alignment that can be reached with a single ultra-short laser pulse is known to be intrinsically limited, recent efforts have concentrated on the possibility of enhancing the total alignment. The maximization of alignment or orientation using a monotonically convergent algorithm has been recently investigated theoretically [10] but the outcome optimal field, found in the microwave part of the spectrum, is rather difficult to implement for practical applications. Experimentally, two alternative approaches have been tested. The first one, combining short and long pulses, has been successfully applied to enhance alignment of iodobenzene molecule [11]. The second one proposed by Leibscher et al. [12] relies on a sequence of several pulses. Such a multipulse strategy is based on optimal control theory in a space of parameters related to the relative peak intensities and delays between the pulses. The dimension of this space is for practical applications rather low due to the small number of pulses that can be reasonably used. It has been shown experimentally that a bi-pulse offers already a significant enhancement of the alignment in asymmetric top [13] and symmetric [14] molecules. An alternative approach consists of designing the spectral phase of a single pulse with a large search space related to the available number of pixels controlling the phase. Recent attempts reported by different groups have revealed the complexity of this task $[8,15]$. The present paper aims at demonstrating the efficiency of such optimizations under realistic conditions of intensity, temperature, and pulse shaping in regard to the potentialities offered by the available pulse shapers. The optimal pulse shapes are synthesized iteratively using an evolutionary algorithm in a feedback loop. The investigation is conducted in $\mathrm{O}_{2}$ because it is a molecule of fundamental interests in the field of molecular alignment and because its rotational constant leads to calculation times suitable with the time-consuming iterative procedure of a learning algorithm. We show theoretically that the field-free molecular alignment at a temperature of $60 \mathrm{~K}$ under constraint of constant energy is significantly enhanced by appropriately manipulating the spectral phase of a femtosecond laser pulse. The mechanism of control is analyzed and the solution reached by the algorithm after the optimization procedure is found to be very close to the target density matrix $[16,17]$. This approach appears superior to the bi-pulse strategy in terms of induced ionization since it requires a lower peak intensity. This property is related to the difference in the control mechanism of both approaches. The phase shaping strategy adapts indeed predominantly the phase of the wavepacket in contrast to the bi-pulse strategy that yields a broader wavepacket.

The complex electric field considered for the pulse inducing the molecular alignment is written as

$$
E(t)=\frac{1}{2 \pi} \int_{-\infty}^{+\infty} \varepsilon(\omega) e^{i \phi(\omega)} e^{-i \omega t} d \omega
$$

where $\phi(\omega)$ denotes the spectral phase and $\varepsilon(\omega)$ the spectral amplitude. $\varepsilon(\omega)$ is taken gaussian with a full width at half maximum associated to a Fourier Transform Limited (FTL) pulse of 100 femtosecond (fs). For concreteness, the spectral phase is controlled, like with the most common spatial light modulator [18], through 128 pixels 
equally distributed across the spectrum, i.e.

$$
\phi(\omega)=\sum_{n=-63}^{n=64} \operatorname{squ}\left(\frac{\omega-\omega_{n}}{\Delta \omega}\right) \phi_{n},
$$

with $\operatorname{squ}(x)=1$ for $|x| \leq 1 / 2$ and $\operatorname{squ}(x)=0$ for $|x|>1 / 2$. $\omega_{n}, \phi_{n}$ stand for the central pulsation and the applied phase of the $n$th pixel while $\Delta \omega$ refers to the spectral sampling, i.e. the pulsation difference between two adjacent pixels $\left(\Delta \omega=0.8 \times 10^{12} \mathrm{rad} / \mathrm{s}\right.$ here $)$. The control is exercised in the present work by modifying the phase $\phi_{n}$ of the 128 pixels independently. These control parameters span over $2 \pi$ and correspond to the genes of an individual for the evolutionary algorithm. Briefly, the algorithm starts with a population of 60 individuals randomly selected. The fitness of each individual is assessed by calculating the molecular alignment produced by the corresponding pulse shape as explained later on. At each new generation, the 10 fittest individuals (i.e. those achieving largest alignment) are cloned and the algorithm generates from these "parents" 50 new individuals or "children" through both crossover and mutation. The mutation procedure is twofold. For half of the children, the phase of very few genes (about 1-2\%) is changed totally randomly while for the others, the phase of about $10 \%$ of the genes is modified by adding a random value within a range that is reduced along the optimization procedure. The algorithm produces thus a new population based on the success of the previous one and iteratively adapt the fitness of the population for the sought after effect. A large variety of pulse shapes can be synthesized with the present pulse shaping technique. Nevertheless, the quality of the desired output waveform can be degraded by the pixelization of the spectral phase shaping. The main effect [19] is to introduce temporal replica with a period of $2 \pi / \Delta \omega=7.8$ ps modulated by a $\operatorname{sinc}(\Delta \omega t / 2)$ envelope corresponding to the Fourier transformation of the top hat pixel shape (defined by the squ function in Eq. (2)). Experimentally, the temporal shaping is governed additionally by a Gaussian envelope due to the spatial beam profile in the phase mask. The product of the Gaussian and sinc terms is known as the "time window".

The molecular alignment is characterized by the quantity $\left\langle\cos ^{2} \theta\right\rangle(t)$ where $\theta$ stands for the angle between the molecular axis and the field polarization. For a given spectral phase $\phi(\omega)$, the pulse intensity envelope is computed using Eq. (1). Considering a molecule initially in a state $\left|J_{0}, M_{0}\right\rangle$ (labelling the rotational and the magnetic quantum number), the wavefunction of the system after the interaction with the field is calculated by solving the time-dependant Schrödinger equation. The quantity $\left\langle\cos ^{2} \theta\right\rangle_{\left|J_{0}, M_{0}\right\rangle}(t)$ is then evaluated and the averaging over the thermal distribution of rotational states is performed to provide $\left\langle\cos ^{2} \theta\right\rangle(t)[3]$. For $\mathbf{O}_{2}$ considered in the present work, only rotational states with odd $J_{0}$ values are populated. A set of rotational states from $J=1$ to 49 in the vibronic ground state of the molecule has been used for the calculation since no excited electronic and vibrational states are resonantly coupled by the field. Transient revivals of molecular alignment are then expected to occur periodically every $t=1 /(8 B c)$ with a full revival period $T_{r}=1 /(2 B c)=11.7 \mathrm{ps}$ (where $B=1.436 \mathrm{~cm}^{-1}$ is the rotational constant for $\left.\mathrm{O}_{2}\right)$. The revival shape depends on the laser excitation and the temperature $T$. In a first trial, the objective predetermined to the algorithm was the optimization of $\left\langle\cos ^{2} \theta\right\rangle$ in $\mathrm{O}_{2}$ for $T=60 \mathrm{~K}$ at a fixed time delay $t=T_{r}$. By fixing the optimization time, we approach at best the most favorable experimental conditions in which the degree of alignment is evaluated at a particular delay by a probe pulse without having to scan the complete transient shape as faced in Ref. [8]. The first full revival time is selected as the optimization target since it corresponds to the replica of the zero delay transient alignment where the pulse shaping is exercised. The intensity used for the FTL pulse of this run (i.e. considering $\phi_{n}=0$ $\forall n)$ is $200 \mathrm{TW} / \mathrm{cm}^{2}$. Typically, the convergence of our algorithm for this purpose has been reached after about 60 generations. The spectral phase together with the pulse shape found out by the algorithm are depicted in Fig. 1(a) and the corresponding induced field-free molecular alignment in Fig. 1(b). The comparison with a FTL pulse is displayed in bottom panels.

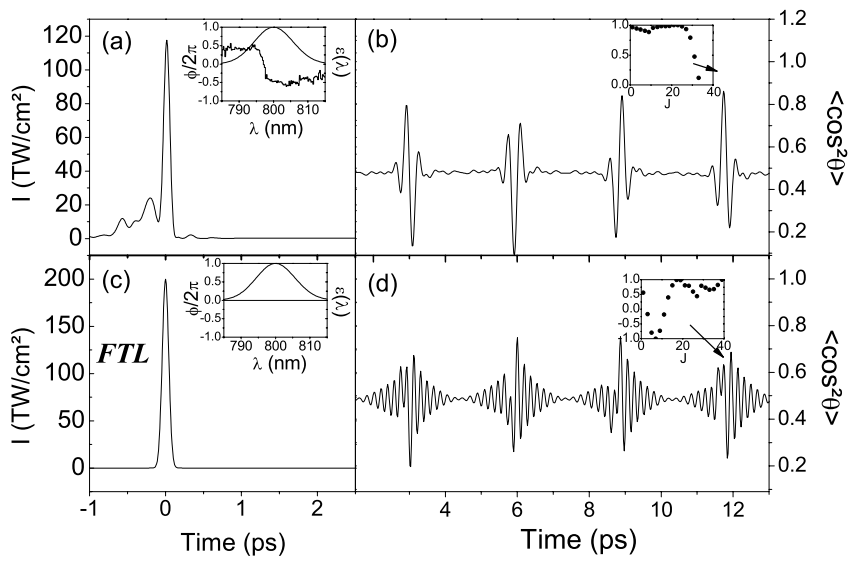

FIG. 1: (a) Optimized pulse shape, (c) FTL pulse with spectral phase and amplitude in insets; (b) and (d) $\left\langle\cos ^{2} \theta\right\rangle(t)$ induced respectively by these two pulse shapes. The insets in (b) and (d) characterize the rephasing of the wavepacket at $t=T_{r}$, i.e. $\cos \left(\omega_{J} T_{r}+\phi_{J}\right)$ vs $J$ (see text).

While the FTL pulse produces a maximum value of $\left\langle\cos ^{2} \theta\right\rangle \approx 0.75$, the phase-shaped pulse reaches a value significantly larger $\left\langle\cos ^{2} \theta\right\rangle \approx 0.86$ with a trace of alignment exhibiting revivals of larger duration. The algorithm succeeds therefore in designing a laser pulse with a better fitness for the issue of alignment. 
Interesting insights can be gained into the effects exercised by the pulse shaping through an analysis in the spectral domain. As explained in Ref. [5], the quantity $\left\langle\cos ^{2} \theta\right\rangle(t)$ can be developed in terms of Fourier components at Raman frequency $\omega_{J}$ [20], i.e. $\left\langle\cos ^{2} \theta\right\rangle(t)=C_{0}+\sum_{J} a_{J} \cos \left(\omega_{J} t+\phi_{J}\right)$. The terms $a_{J}>0$ and $\phi_{J}$ correspond respectively to the amplitude and the phase of the Fourier components that are displayed in Fig. 2 for both FTL and shaped pulses of this investigation. One noticeable feature is the quasi linear phase $\phi_{J}$ as outcome of the optimization. This peculiar result can be interpreted as follow. The alignment at the full revival $t=T_{r}$ is maximized when $\cos \left(\omega_{J} T_{r}+\phi_{J}\right)=1 \forall J$ and thus for a phase $\phi_{J}=-\omega_{J} T_{r}+k 2 \pi=-2 \pi c B T_{r}(4 J+6)+k 2 \pi$, with $k$ an integer, if the centrifugal distorsion can be neglected. It corresponds thus to a linear dependence of $\phi_{J}$ vs $J$ with a negative slope. The latter is shown as a full curve in Fig. 2(b) and is fulfilled to a good approximation, for significant values of $a_{J}$ (i.e. $J \lesssim 25$ ), by the phase distribution $\phi_{J}$ resulting from the excitation with the optimized pulse shape. Based on this interpretation,

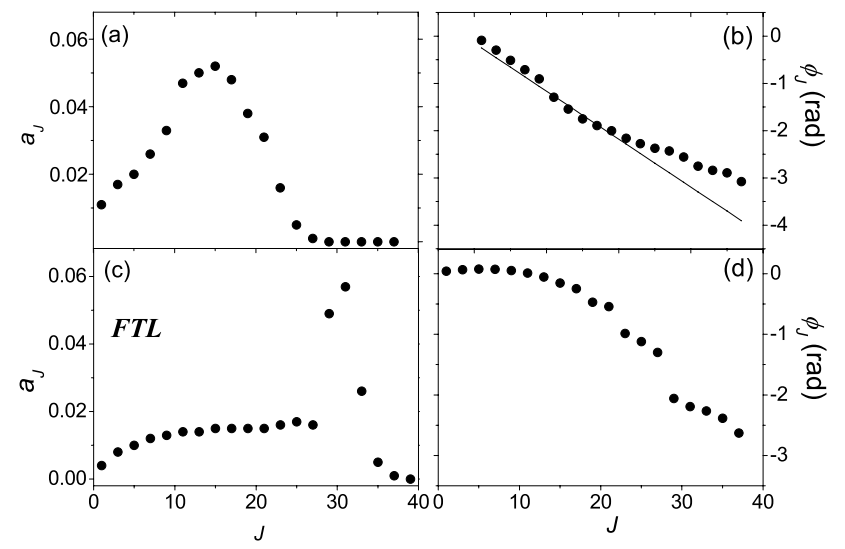

FIG. 2: (a) amplitude $a_{J}$ and (b) phase $\phi_{J}$ vs $J$ of the Fourier components at Raman frequency of $\left\langle\cos ^{2} \theta\right\rangle(t)$ for the optimized pulse shape. (c) and (d) same as (a) and (b) for the FTL pulse. The full curve in panel (b) refers to the criteria of complete rephasing of the rotational wavepacket (see text).

all the Raman components $\omega_{J}$ should achieve a quite complete rephasing at $t=T_{r}$. The latter is confirmed by the inset of Fig. 1(b) displaying the value $\cos \left(\omega_{J} T_{r}+\phi_{J}\right)$ vs $J$. The comparison with the FTL pulse (Fig. 1(d)) is remarkable. While for the FTL pulse, the different components exhibit significant dephasing, the algorithm succeeds in designing pulse shape that plays on the relative timing between them to lead in a quasi-perfect synchronization at $t=T_{r}$. The inspection of the amplitudes $a_{J}$ [Figs. 2(a) and (c)] provides further indications as well. A comparison between the FTL and the shaped pulse reveals that the latter tends to promote the excita- tion of the lower $J$ levels. We argue that the algorithm guides the excitation towards this solution because the low $J$ levels are intrinsically easier to rephase due to their lower energy. One longstanding question in the field of control by feedback-optimized shaped pulses concerns the occurrence of the "optimal solution". To address this question, we have followed the procedure described in Refs. [16, 17], where a target density matrix has been defined. It has been shown that the target density matrix provides the optimum alignment that can be reached for a given reduced Hilbert space (defined by the states significantly populated during the interaction). We have analyzed the wavepacket resulting from the optimization at $t=T_{r}$ and the outcome is the achievement of $86 \%$ of the target density matrix with a degree of alignment around $97 \%$ of the latter. In view of the limitation of the search space, this is a compelling result. It should be noted furthermore that the maximum peak intensity of the tailored pulse (Fig. 1(a)) has been reduced to about $115 \mathrm{TW} / \mathrm{cm}^{2}$ due to the energy-constant pulse shaping. This aspect is crucial in regard to ionization. A rough estimation of the ionization probability following the model of Ref. [21] yields a value $P_{\text {ion }} \approx 0.36$ for the shaped pulse drastically reduced compared to the FTL pulse for which the ionization is almost saturated $\left(P_{\text {ion }} \approx 0.99\right)$. These values are of great importance for an experimental demonstration and the high ionization induced by the FTL pulse is expected to strengthen the enhancement of alignment observed in Fig. 1 for the shaped pulse compared with the FTL pulse. A set of runs performed in similar conditions has confirmed the general features of the present optimization reflecting its robustness. Other different FTL peak intensities from 50 to $300 \mathrm{TW} / \mathrm{cm}^{2}$ have been investigated as well. In particular, for a peak intensity of $50 \mathrm{TW} / \mathrm{cm}^{2}$ the algorithm has converged towards the FTL pulse shape and did not succeed in enhancing the alignment. Our investigation confirms thus the general idea that the optimization likely occurs for intensities where the alignment by a FTL pulse is saturated. At $T=60 \mathrm{~K}$ the saturation of the alignment occurs around $100 \mathrm{TW} / \mathrm{cm}^{2}$ vs $200 \mathrm{TW} / \mathrm{cm}^{2}$ for $T=300 \mathrm{~K}$. While the optimization at room temperature (attempted in Ref. [8]) is in principle possible, the experimental observation would nevertheless require larger intensity giving rise to important ionization. For $T=60 \mathrm{~K}$, the algorithm did optimize the alignment for all intensities $I \geqslant 100 \mathrm{TW} / \mathrm{cm}^{2}$. Corresponding optimized pulse shapes all exhibit similar features, i.e. a long leading edge with several subpeaks prior to a major pulse centered at $t=0$ (Fig. 1(a)). Although some changes particularly in the leading edge make each pulse specific with respect to the peak intensity, the result is found to be rather robust to the spatial averaging. The recurrent temporal structures observed in the optimized pulse shapes should shed light on the control mechanism. The analysis of the dynamical alignment reveals that the long tail induces a prealignment that reaches its 
maximum around $t=0$ where the field presents its maximum intensity. The underlying mechanism exhibits thus analogy with the multipulse strategy proposed in Ref.[12] and reported experimentally [13, 14] using a sequence of two pulses. We have compared the possibilities offered by both approaches. The alignment with a bi-pulse in the conditions of temperature and energy of this work is found numerically maximized for a sequence of pulses of intensities $I=40-160 \mathrm{TW} / \mathrm{cm}^{2}$ time-delayed by about $3050 \mathrm{fs}$. The maximum alignment $\left(\left\langle\cos ^{2} \theta\right\rangle \approx\right.$ $0.86)$ is similar to the one obtained with the optimized phase-shaped laser pulse. Although the global alignment is comparable, the control exercised by the bi-pulse strategy is nevertheless different and less attractive as shown below. The optimized pulse shape leads to a wavepacket with precisely adapted phase, while the bi-pulse favors the creation of a broader wavepacket in the $J$-space at the cost of lower rephasing quality. Since the fitness (i.e. the maximum value of $\left\langle\cos ^{2} \theta\right\rangle$ ) reached by both methods is the same, it can not be excluded a priori that the algorithm could recover the bi-pulse solution. We argue that the constraints of the present pulse shaping are unfavorable to a bi-pulse strategy. When using a realistic description of the pixel shape, the pulse shaping with large extend (out to $3 \mathrm{ps}$ ) is indeed limited by the time-window and inherent replica whereas tailoring located near zero time exhibits a better fidelity. For these reasons, the algorithm has converged towards an alternative pulse shape that is better suited with the 128-pixels control. Although the resulting maximum alignment is similar, the present phase shaping features a crucial benefit. In order to reach the same degree of alignment, the bi-pulse approach requires a large peak intensity, i.e. $160 \mathrm{TW} / \mathrm{cm}^{2}$ vs $115 \mathrm{TW} / \mathrm{cm}^{2}$ for the optimized pulse, leading to a high ionization probability $\left(P_{\text {ion }} \approx 0.85\right)$. This makes clearly the phase shaping technique superior. The multipulse strategy should imply more than two pulses to reduce the ionization and appears then more complicated to implement experimentally.
To conclude, we have investigated the optimization of postpulse molecular alignment in $\mathrm{O}_{2}$ by means of phase-shaped fs laser pulses. The alignment at $T=60 \mathrm{~K}$ is shown significantly enhanced using a specific laser pulse shape designed by an evolutionary algorithm. While a bi-pulse strategy is expected to produce similar global alignment, the solution found out by the present approach appears superior in terms of peak intensity and induced ionization. Further works at $T=100 \mathrm{~K}$ as well as in other molecules $\left(\mathrm{N}_{2}\right.$ and $\left.\mathrm{CO}_{2}\right)$ have corroborated these results. Based on these findings, the phase-shaping of fs laser pulses by self learning algorithms turns out to be a valuable alternative approach to the multipulse strategy. Optimally shaped laser pulses features promising applications in the frame of alignment. As suggested in Ref. [22], the postpulse molecular alignment can be exploited in order to achieve isotopic separation. The basic idea is to provide at a specific time two molecular isotopes with drastically different angular distributions (i.e. one aligned with the other one delocalized) as a result of the slight difference in their rotational constants. The physical separation is then realized for instance through angular-dependant ionization or dissociation processes. The selectivity of the method can be described in some cases as the ratio of $\left\langle\cos ^{2} \theta\right\rangle$ associated to each isotope. Using the present optimization procedure, we have been able to improve significantly the latter. This potentiality will be investigated in a future publication.

\section{Acknowledgments}

The authors acknowledge D. Sugny for fruitfull discussions. This work was supported by the Conseil Régional de Bourgogne, the CNRS, the ACI "Photonique", and a Marie Curie European Reintegration Grant within the 6th European Community RTD Framework Programme.
[1] H. Stapelfeldt and T. Seideman, Rev. Mod. Phys. 75 (2), 543 (2003).

[2] F. Rosca-Pruna and M. J. J. Vrakking, Phys. Rev. Lett. 87 (15), 153902 (2001).

[3] V. Renard, M. Renard, S. Guérin, Y. T. Pashayan, B. Lavorel, O. Faucher, and H. R. Jauslin, Phys. Rev. Lett. 90 (15), 153601 (2003).

[4] P. W. Dooley, I. V. Litvinyuk, K. F. Lee, D. M. Rayner, M. Spanner, D. M. Villeneuve, and P. B. Corkum, Phys. Rev. A 68 (2), 023406 (2003).

[5] M. Renard, E. Hertz, S. Guérin, H. R. Jauslin, B. Lavorel, and O. Faucher, Phys. Rev. A 72, 025401 (2005).

[6] M. Spanner, E. A. Shapiro, and M. Ivanov, Phys. Rev. Lett. 92, 093001 (2004).

[7] M. Renard, E. Hertz, B. Lavorel, and O. Faucher, Phys. Rev. A 69, 043401 (2004).
[8] C. Horn, M. Wollenhaupt, M. Krug, T. Baumert, R. de Nalda, and L. Bañares, Phys. Rev. A 73, 031401(R) (2006).

[9] D. Daems, S. Guérin, E. Hertz, H. R. Jauslin, B. Lavorel, and O. Faucher, Phys. Rev. Lett. 95, 063005 (2005).

[10] J. Salomon, C. M. Dion, G. Turinici, J. Chem. Phys. 123, 144310 (2005).

[11] M.D. Poulsen, T. Ejdrup, H. Stapelfeldt, E. Hamilton, T. Seideman, Phys. Rev. A 73, 033405 (2006).

[12] M. Leibscher, I. Sh. Averbukh, and H. Rabitz, Phys. Rev. Lett. 90, 213001 (2003).

[13] C. Z. Bisgaard, M. D. Poulsen, E. Péronne, S. S. Viftrup, and H. Stapelfeldt, Phys. Rev. Lett. 92, 173004 (2004).

[14] C. Z. Bisgaard, S. S. Viftrup, and H. Stapelfeldt, Phys. Rev. A 73, 053410 (2006).

[15] Ch. Siedschlag, O.M. Shir, Th. Bäck, M.J.J. Vrakking, 
Opt. Comm. 264, 511 (2006).

[16] D. Sugny, A. Keller, O. Atabek, D. Daems, C. M. Dion, S. Guérin, and H. R. Jauslin, Phys. Rev. A 71, 063402 (2005).

[17] D. Sugny, A. Keller, O. Atabek, D. Daems, C. M. Dion, S. Guérin, and H. R. Jauslin, Phys. Rev. A 73, 032704 (2005).

[18] A. M. Weiner, Rev. Sci. Instrum. 71, 1929 (2000).

[19] J. C. Vaughan, T. Feurer, K. W. Stone, K. A. Nelson,
Opt. Express 14, $1314(2006)$

$[20] \omega_{J}=2 \pi c\left[B(4 J+6)-D\left((J+2)^{2}(J+3)^{2}-J^{2}(J+1)^{2}\right)\right]$ with $D$ the centrifugal distorsion.

[21] A. Talepbour, J. Yang, and S. L. Chin, Opt. Comm. 163, 29 (1999).

[22] S. Fleischer, I. Sh. Averbukh, and Y. Prior, Phys. Rev. A 74, 041403(R) (2006). 